\title{
An interactive toolbox for atlas-based segmentation and coding of volumetric images
}

\author{
G. Menegaz ${ }^{1}$, S.Luti ${ }^{1}$, V. Duay ${ }^{2}$, J.-Ph. Thiran² \\ ${ }^{1}$ Dept. of Information Engineering, University of Siena, Italy \\ 2 Signal Processing Institute, EPFL, Lausanne, Switzerland
}

\begin{abstract}
Medical imaging poses the great challenge of having compression algorithms that are lossless for diagnostic and legal reasons and yet provide high compression rates for reduced storage and transmission time. The images usually consist of a region of interest representing the part of the body under investigation surrounded by a "background", which is often noisy and not of diagnostic interest. In this paper, we propose a ROI-based 3D coding system integrating both the segmentation and the compression tools. The ROI is extracted by an atlas based 3D segmentation method combining active contours with information theoretic principles, and the resulting segmentation map is exploited for ROI based coding. The system is equipped with a GUI allowing the medical doctors to supervise the segmentation process and eventually reshape the detected contours at any point. The process is initiated by the user through the selection of either one pre-defined reference image or one image of the volume to be used as the 2D "atlas". The object contour is successively propagated from one frame to the next where it is used as the initial border estimation. In this way, the entire volume is segmented based on a unique $2 \mathrm{D}$ atlas. The resulting $3 \mathrm{D}$ segmentation map is exploited for adaptive coding of the different image regions. Two coding systems were considered: the JPEG3D standard and the 3D-SPITH. The evaluation of the performance with respect to both segmentation and coding proved the high potential of the proposed system in providing an integrated, low-cost and computationally effective solution for CAD and PAC systems.
\end{abstract}

Keywords: medical image segmentation, volumetric compression, JP3D.

\section{INTRODUCTION}

Medical data are increasingly represented as volumes. The efficient storage and transmission of such data rises the issue of medical image coding. Indeed, the ISO/IEC is currently finalizing a standardized solution for volumetric data compression to be enclosed in the JPEG2000 standard (JP3D, Part 10). Here we propose an $a d$-hoc system integrating both the automatic segmentation and the ROI-based coding of volumetric data. Medical images, in fact, usually consist of a region of interest representing the part of the body under investigation (i.e. the heart in a chest CT or MRI scan, the brain in a head MRI scan) surrounded by background, often noisy, with no diagnostic interest. It seems thus very natural to process such data in an object-based framework: assign higher priority to the semantically relevant object, to be represented with up-to lossless quality, and lower priority to the background, for which the lossless constraint can be relaxed.

The proposed system consists of two main building blocks: the segmentation and the compression tools.

The segmentation method is inspired to the approach proposed in $^{1}$ and will be presented in detail in a joint paper. $^{2}$ The basic idea consists in integrating the a-priori information about the data brought by a reference image (the atlas) within an active contours based framework. Both boundary and region information are accounted for and exploited to make an initial contour evolve toward the edge of the object of interest. The role of the atlas depends on the choice of the region based term, as will be detailed in the next Section.

In this work such an approach is generalized for the segmentation of volumetric data. Each image of the stack can play the role of target and atlas image, respectively, during the segmentation process. Among the main

Further author information: (Send correspondence to Gloria Menegaz)

Gloria Menegaz: E-mail: gloria@ieee.org, Telephone: (+39) 3472248704

Address: Via Roma 56, I-53100 Siena, Italy 
features of the resulting volumetric segmentation technique are the following: (i) a single atlas image is sufficient to drive the process towards the desired solution; (ii) the atlas image can be either one image of the dataset or a predefined reference image (depending on the context); (iii) in the worst case, only one image of the set needs to be segmented by hand. Furthermore, following the requirements of the medical community, the segmentation tool has been equipped with a graphical user interface (GUI) enabling the medical doctor to supervise the process and eventually reshape the detected contour at any point. The so-identified ROI is exploited for object-based coding. Two state-of-the-art fully 3D coding system were considered: the novel JP3D algorithm and 3D-SPIHT.

This paper is organized as follows. Section 2 illustrates the segmentation methods; in particular, Section 2.1 summarizes the different segmentation techniques and Section 2.2 describes their generalization to the threedimensional case as well as the GUI; Section 3 illustrates the results and Section 4 derives conclusions.

\section{METHODS}

\subsection{Atlas-based active contours}

The proposed volumetric segmentation technique grounds on the atlas-based active contours paradigm that has been designed and implemented for the 2D case by the authors. Without going into the details of such an approach, that will be extensively illustrated in a joint work, ${ }^{?}$ the basic ideas and concepts will be summarized hereafter. The proposed segmentation method is a variation on the theme of the active contours in the generalized form accounting for both a boundary and a region-dependent term in the evolution equation. ${ }^{1}$ The basic idea of active contours is to identify a target function in the form of a partial differential equation that makes an initial estimate of the wanted contour evolve (following the minimization of the corresponding error criterion) such that it will converge to the actual boundary separating the two regions. Accounting for both the boundary and the textural features leads to a functional of the form

$$
J\left(\Omega_{\text {in }}, \Omega_{\text {out }}, \Gamma\right)=\int_{\Omega_{\text {in }}} k_{\text {in }}\left(\vec{x}, \Omega_{\text {in }}\right) d \vec{x}+\int_{\Omega_{\text {out }}} k_{\text {out }}\left(\vec{x}, \Omega_{\text {out }}\right) d \vec{x}+\int_{\Gamma} k_{b}(\vec{x}) d \vec{a}(\vec{x})
$$

where $\Omega_{\text {in }}$ and $\Omega_{\text {out }}$ denote the regions inside and outside of the contour, respectively, $k_{\tau}\left(\vec{x}, \Omega_{\tau}\right), \tau=i n$, out are the region-based terms, and $k_{b}(\vec{x})$ is the boundary term. Following the definition of the shape gradient, ${ }^{1}$ the choice of the region-based terms will specify the evolution equation, given a pre-defined boundary term. In particular, the derivation of the evolution equation for a region-dependent descriptor passes through the computation of the Eulerian derivative of the corresponding functional in the direction of $\vec{V}$, namely the velocity vector field as defined in: ${ }^{1}$

$$
d J_{r}(\Omega, \vec{V})=-\int_{\Gamma}(A(\vec{x}, \Omega)+k(\vec{x}, \Omega))(\vec{V} \cdot \vec{N}) d \vec{a}(\vec{x})
$$

where $\vec{N}$ is the inward normal to $\Gamma$ and $d \vec{a}$ is the area element. In our work, we have focused on the particular case in which the region descriptor is based on the entropy. Two cases were considered: (i) each of the two regions is described by the respective marginal entropy; (ii) the joint entropy of the two regions in different image instances is used as the criterion to drive the evolution equation. The novelty of the proposed approach consists in introducing the a-priori information brought by the atlas to improve the performance in terms of both the "quality" of the segmentation and the computational efficiency. However, the original method using the marginal entropy in the region competition paradigm as in ${ }^{1}$ will be used for benchmarking and labeled as marginal entropy (ME)-based method. Similarly, the novel version using the atlas will be referred to as atlasbased marginal entropy ( $A B M E$ ) method, while the last one exploiting the joint entropy will be called atlas-based joint entropy (ABJE) method.

\subsubsection{Marginal Entropy (ME)}

Talking about marginal entropy implies the region competition paradigm between $\Omega_{\text {in }}$ and $\Omega_{\text {out }}$ sharing the boundary $\Gamma$. In this case the functional to minimize is

$$
J\left(\Omega_{\text {in }}, \Omega_{\text {out }}, \Gamma\right)=H\left(\Omega_{\text {in }}\right)+H\left(\Omega_{\text {out }}\right)+\int_{\Gamma} \lambda d \vec{a}(\vec{x})
$$


where $\lambda$ is a regularization parameter. The entropy based descriptors are

$$
H\left(\Omega_{\nu}\right)=\int_{\Omega_{\nu}}-q\left(f\left(\vec{x}, \Omega_{\nu}\right)\right) \ln q\left(f\left(\vec{x}, \Omega_{\nu}\right)\right) d \vec{x}
$$

where $\nu=i n$, out and

$$
k(\vec{x}, \Omega)=-q\left(f\left(\vec{x}, \Omega_{\nu}\right)\right) \ln q\left(f\left(\vec{x}, \Omega_{\nu}\right)\right)
$$

The corresponding evolution term is

$$
\begin{aligned}
& \frac{\partial \Gamma}{\partial \tau}(\vec{x})=\left(\left(A\left(\vec{x}, \Omega_{\text {in }}\right)+\varphi\left(q\left(f\left(\vec{x}, \Omega_{\text {in }}\right)\right)\right)+\left(A\left(\vec{x}, \Omega_{\text {out }}\right)+\varphi\left(q\left(f\left(\vec{x}, \Omega_{\text {out }}\right)\right)\right)+\lambda \kappa\right) \vec{N}\right.\right. \\
& \varphi\left(q\left(f(\vec{x}), \Omega_{\nu}\right)\right)=-q\left(f\left(\vec{x}, \Omega_{\nu}\right)\right) \ln q\left(f\left(\vec{x}, \Omega_{\nu}\right)\right)
\end{aligned}
$$

where $\kappa$ is the curvature of $\Gamma$. The ME method presents two main disadvantages. First, it is only suitable for the segregation of homogeneous regions. The minimization of the entropies of the two competing parts of the image implies the assumption of homogeneity and the accuracy strongly depends on the distance between the respective histograms. Second, the minimization of the objective function is computationally expensive because at each iteration the marginal histograms and the entropies of the competing regions need to be computed. In order to overcome these limitations, the ABME and the ABJE methods were devised and implemented.

\subsubsection{Atlas-based marginal entropy (ABME)}

The ABME method rests on the assumption that, given a reference image (the atlas) whose region-based features are sufficiently close to those of the image to segment (the target), the computation of the region descriptors can be removed from the iteration loop and performed only once at the beginning of the process on the atlas image. Basically, this amounts to assume that the probability density functions for the regions $\Omega_{\text {in }}$ and $\Omega_{\text {out }}$ as estimated on the atlas image are well representative of those of the corresponding regions on the target. Accordingly, they are evaluated off-line as a preprocessing step and used at each iteration of the minimization loop for the computation of the marginal entropies of the regions, as required by (6). This substantially reduces the complexity making the algorithm more attractive for real time applications. However, the constraint on the homogeneity of the competing regions still holds. Results show that the performance of the ABME algorithm are strongly dependent on the proximity of the pdfs of $\Omega_{\text {in }}$ and $\Omega_{\text {out }}$ when evaluated on the atlas and on the target, respectively.

\subsubsection{Atlas-based Joint Entropy (ABJE)}

The minimization of the joint entropy has extensively been used for image registration. The use of the joint entropy for the segmentation of vectorial images has been proposed in. ${ }^{1}$ The corresponding objective function to minimize is

$$
J\left(\Omega_{\text {in }}, \Omega_{\text {out }}, \Gamma\right)=H_{X Y}\left(\Omega_{\text {in }}\right)+H_{X Y}\left(\Omega_{\text {out }}\right)+\int_{\Gamma} \lambda d \vec{a}(\vec{x})
$$

where

$$
H_{X Y}\left(\Omega_{X}, \Omega_{Y}\right)=-\int_{\Omega_{X}} \int_{\Omega_{Y}} q_{X Y}(x, y) \ln q_{X Y}(x, y) d x d y
$$

where $\Omega_{X}, \Omega_{Y}$ are the sets of the possible outcomes of $X$ and $Y$, respectively. In the reference work, ${ }^{1}$ this approach was exploited for the segmentation of multi-channel (color) images. The corresponding evolution equation is

$$
\frac{\partial \Gamma}{\partial \tau}(\vec{x})=\left(\left(A\left(\vec{x}, \Omega_{\text {in }}\right)+\varphi\left(q\left(\vec{f}(\vec{x}), \Omega_{\text {in }}\right)\right)\right)-\left(A\left(\vec{x}, \Omega_{\text {out }}\right)+\varphi\left(q\left(\vec{f}(\vec{x}), \Omega_{\text {out }}\right)\right)\right)+\lambda \kappa\right) \vec{N}
$$

where $A(\vec{x}, \Omega)$ is the region dependent term which is a function of the joint entropy. Again, the main bottleneck is computational load since the joint pdfs are estimated at each iteration. However, robustness is improved with respect to both $\mathrm{ME}$ and $\mathrm{ABME}$ methods as well as the performance due to the relaxation of the homogeneity constraint. 


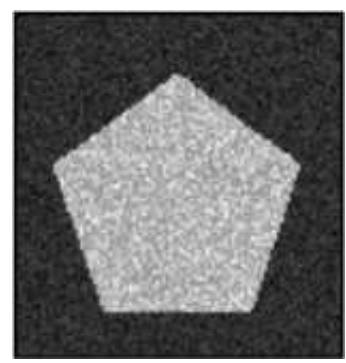

(a)

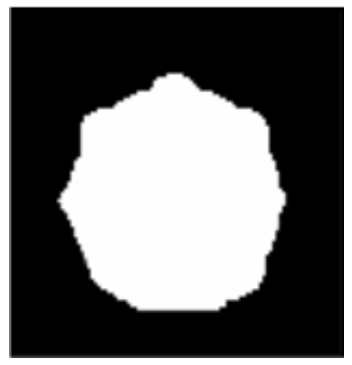

(d)

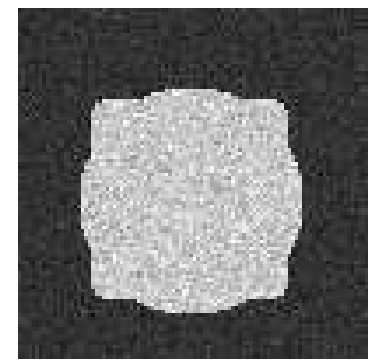

(b)

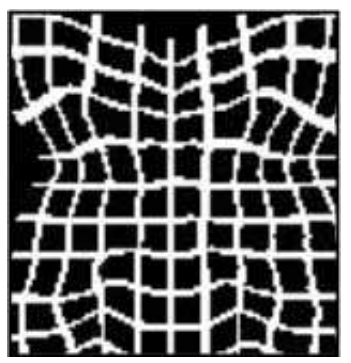

(e)

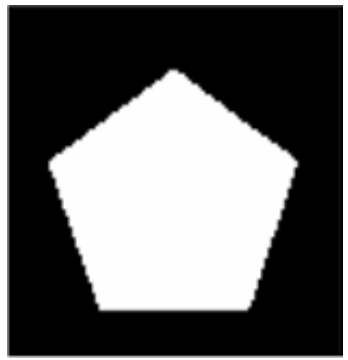

(c)

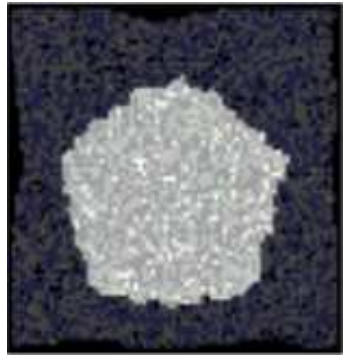

(f)

Figure 1. (a) Atlas; (b) Target; (c) Atlas mask; (d) Current boundary; (e) Deformation field (f) Deformed atlas.

In the proposed paradigm, the atlas and the target images are used instead of the different color channels of a multispectral image. The joint entropy between the atlas and the target image is the driving force of the contour during the segmentation process. The basic idea is that the minimization of the joint entropy will lead the initial contour, specified by the atlas, to converge to the actual boundary between the competing regions. At that point, the segmentation of the target region results from the projection of the region-specific labels from the atlas to the target image. Figure (1) illustrates the algorithm using a synthetic image. Given the atlas image (Figure 1(a)), a mask is derived associating a different color code to the two regions (Figure 1(c)). The atlas is segmented by hand or a previous segmentation is assumed to be available. Then, the minimization loop is started taking the mask boundary as the initial estimate of the contour. From then on, at each iteration, the following operations are performed:

1. The atlas mask is deformed according to the current estimate of the contour (Figure 1(d));

2. The resulting deformation field (Figure 1(e)) is applied to the atlas image (Figure 1(f));

3. The joint histogram between the deformed atlas image and the target image is computed;

4. The joint entropy is computed and plugged into the evolution equation.

The Parzen window method is used for the estimation of the joint pdf. This allows to smooth out the joint histogram avoiding sharp peaks that could drive the optimization to local minima.

\subsection{Generalization for volumetric data}

Each of the segmentation methods introduced in the previous Section was generalized for the manipulation of 3D data distributions. The 3D extension of the ME method was used for benchmarking.

The common ground on which the different applications were built is the idea to exploit the similarity between adjacent images by propagating the segmentation control parameters from one image to the next. In particular, during the segmentation of the image $n$ the role of the atlas is played by the image segmented in the previous step, namely image $n-1$. In practice, after the segmentation of slice $n$, this becomes the atlas to be used for 
the segmentation of the slice $n+1$, which corresponds to the current target image. The region descriptor is updated accordingly to start the segmentation process on the current image. A reference image is used as the initial atlas. This could be either one image of the actual dataset of the patient under clinical investigation or a pre-defined one. Fig. (2) illustrates the procedure.

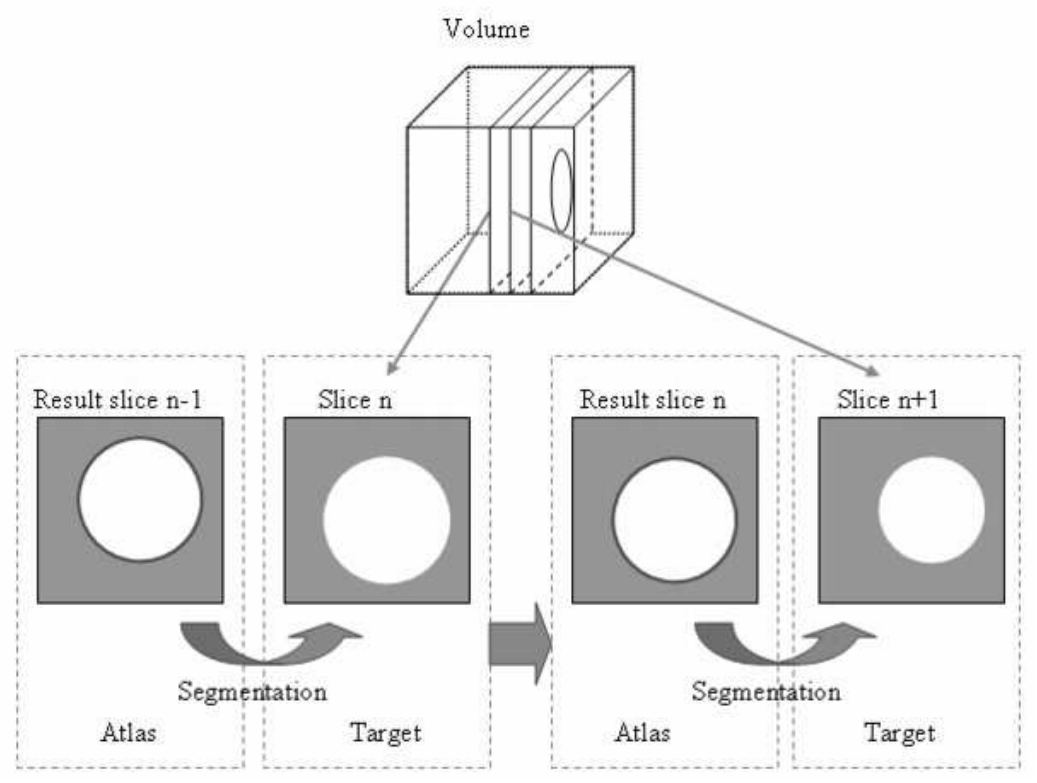

Figure 2. Extension to volumetric data. After the segmentation of slice $n$, this becomes the atlas to be used for the segmentation of the slice $n+1$, i.e. the current target image.

User interaction is enabled through the GUI such that the whole set of parameters can be re-initialized at any point during the segmentation process. This includes both the current boundary and the atlas image.

Marginal Entropy The 3D extension of the ME method was obtained by separately segmenting all the images of the dataset. The difference with respect to the $2 \mathrm{D}$ case is in the initialization: the contour detected in the previous image is used to start the process in the next one.

Atlas-based Marginal Entropy The marginal histograms are evaluated on the intial atlas as a pre-processing step and dynamically updated during the segmentation process. Again, the contour is propagated along the $z$ axis for initialization purposes. Accordingly, only one image eventually needs to be manually segmented, avoiding the need to provide an hand made segmentation of the entire volume for initialization. As mentioned above, the GUI allows to dynamically adapt both the boundary and the atlas. This improves the accuracy at the expenses of computational complexity.

Atlas-based Joint Entropy For the ABJE method, the joint entropy between the atlas and the target is dynamically updated. As was the case in $2 \mathrm{D}$, this method provides a clear advantage over the others concerning segmentation accuracy and robustness, at the expense of an increased computational complexity. Again, the segmentation parameters can be manually adjusted at any time through the GUI.

\subsubsection{The graphical user interface}

Even though, in general, unsupervised segmentation is desirable, this does not hold in the medical field, where doctors prefer to maintain the control over the process and the ability to interact with the software at any point 
to provide their feedback and eventually reshape the solution on the fly. To cope with this requirement, the system is equipped with a GUI where the segmentation result is progressively displayed. A stop-button allows to interrupt the process and start the manual reshaping of the region. A new window is open where the new contour can me drawn by hand and used in the next steps. This also avoids error propagation. Given the sensitivity of the proposed methods to the accuracy of the atlas, this kind of errors can easily be present and the supervision allows to keep them under control. The configuration parameters are written in a text file indicating (i) the name and location of the atlas image; (ii) the related mask; (iii) the index of the first slice from where to start the segmentation within the volume; (iv) the number of images composing the volume.

The GUI provides also a configuration tool enabling to select the segmentation method among the different possibilities (marginal entropy atlas-based or joint entropy) as well as the parameters that the chosen method needs. In this way, not only the contour but also the segmentation parameters can be changed during the segmentation to dynamically adapt the process to the desired results.

\subsection{D ROI-based Coding}

The segmentation map is used to select the ROI. Two conding engine were considered: the standard JP3D (JPEG part 10) verification model as implemented in, ${ }^{3}$ and the $3 \mathrm{D}-\mathrm{SPIHT}^{4}$ as implemented in. ${ }^{5}$ Since the JP3D does not feature ROI based functionalities, such modality was emulated by setting to zero all the voxels outside the region being encoded. Only two regions (the object and the background) were considered for simplicity, each corresponding to one segment of the bit stream, ensuring random access to the object of interest that can be decoded at the desired quality. In both cases, integer lifting was used for the implementation of the discrete wavelet transform to enable the lossless functionality. The $5 \times 3$ interpolating filter ${ }^{6}$ was used. Figure (3) provides an illustration.

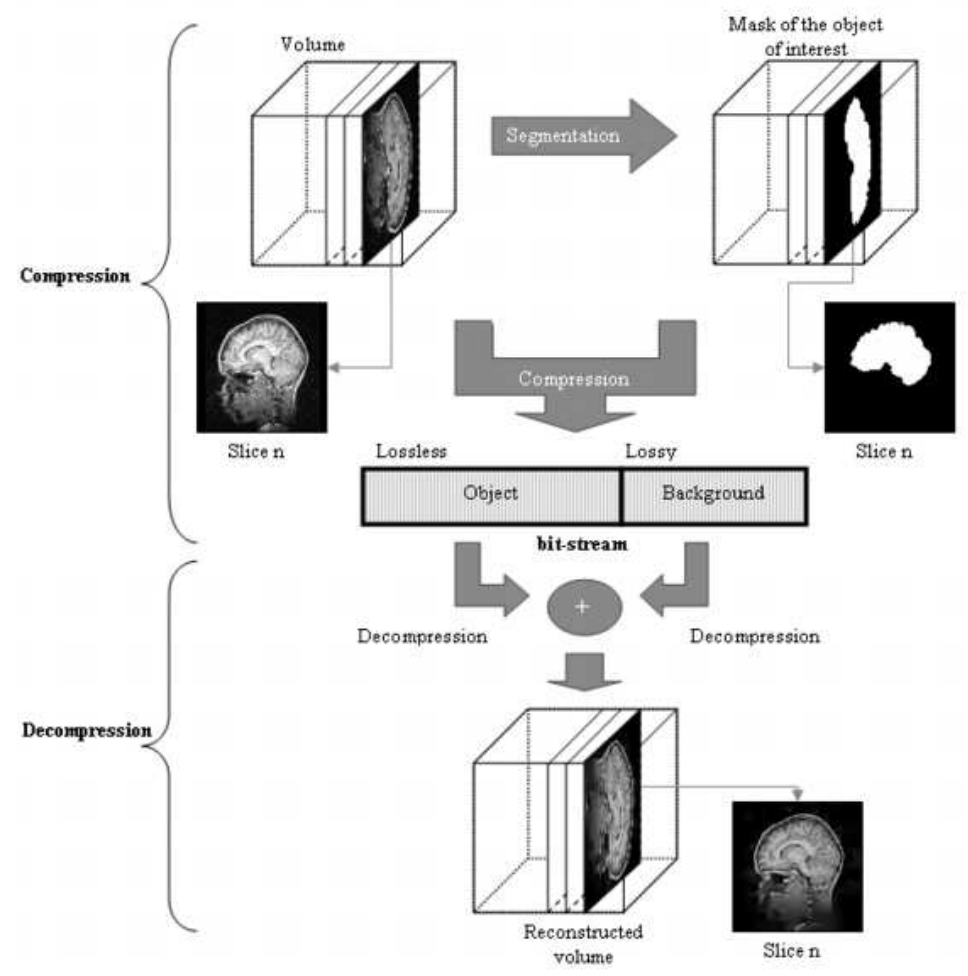

Figure 3. ROI-based coding system. 


\section{RESULTS AND DISCUSSION}

\subsection{Volume Segmentation}

The proposed methods were applied to the segmentation of two head MRI datasets.

\subsubsection{Dataset 1}

This consists of256x256x128 voxels. The central image along the $z$ axis was chosen as the atlas and segmented by hand. Figures 4 illustrates the results. The ABME method has proven to be the most suitable for this kind of data. The average computational time for each slice was 7,4 sec. In order to reach an accurate segmentation, human interaction was required to suitably account for the changes in the internal structures of the brain. In the example the re-initialization was performed eight times. The ABJE method, instead, is much more computationally expensive but provides most accurate results, as expected. In this case, the segmentation of each slice took $300 \mathrm{sec}$ while the segmentation of the entire volume needed almost 10 hours with supervision. The process was re-initialized seven times.

\subsubsection{Dataset 2}

The volume size is $256 \times 256 \times 124$ voxels. The reference image to be used as the atlas was taken from the dataset pertaining to a different patient and was segmented by hand. Then, it was used to initialize the segmentation process starting at the central slice in the volume. The atlas and the starting image are shown in Figure 5 . The proposed 3D segmentation tool was then used to propagate the contour of the object of interest upwards and downwards in the volume. Results demonstrate that it is possible to use a unique atlas for a group of volumes that feature the same structures and objects. Figure 6 shows the results for the ABME and ABJE methods. As was the case for Dataset 1, the ABME provides the best performance. The time taken for the segmentation is of the same order of magnitude as for Dataset 1 in both cases. Six and seven re-initializations were needed for $\mathrm{ABME}$ and ABJE, respectively.

\subsection{Coding}

Here we analyze the performances of the JP3D against the 3D-SPHIT algorithm. In the ROI compression the accuracy of the mask does not significantly affect the coding gain. However, it has an impact on the perceived quality of the reconstructed image. The segmentation mask provided by the ABJE method was used. The PSNR was used as objective quality metric. In the 3D case the PSNR changes image-wise. In order to have a global measure for the distortion of the entire reconstructed volume we consider the average PSNR along the $z$ axis. This was computed by calculating the average value for the MSE and then computing the PSNR. The ROIbased functionality was implemented by setting to zero all the voxels outside and inside the ROI, respectively, for generating the bitstream segments pertaining to the region of interest and the background, respectively. The integer version of the Cohen-Daubechies-Feauveau $5 \times 3$ filter was used. The depth of the DWT was set to 5 .

For Dataset 1 the lossless rates on the entire volume were 3.59 and $3.96 \mathrm{bpv}$ for JP3D and 3D-SPIHT, respectively. The performance in lossy mode on the entire volume was characterized by the rate-distortion curve as well as by the evaluation of the PSNR as a function of the slice index along the $z$ axis for a given decoding rate $(0.5 \mathrm{bpv})$. Figure 7 shows the results. Results show that JP3D outperforms SPIHT at medium to high rates. The SPIHT algorithm is affected by the characteristic oscillation of the PSNR with the slice index due to the rounding noise introduced by integer lifting. ${ }^{7,8}$ Conversely JPEG2000 does not suffer from such shortcoming due to the post-compression rate distortion policy.

ROI functionalities enable the independent encoding/decoding of the object and the background such that they can be accessed and recovered at the desired rate. Lossless performance is unavoidable in the ROI. The lossless rates provided by the JP3D and 3D-SPIHT algorithms for the ROI amount to 1.11 and $1.32 \mathrm{bpv}$, respectively. The hybrid semantically lossless mode can be reached by the lossless representation of the ROI and the lossy coding of the background. This allows to assign the available transmission/storage resources to the diagnostically relevant part of the data. Furthermore, both the systems feature scalability functionalities.

In the ROI based mode, JP3D outperforms 3D-SPIHT providing a lower lossless rate. Accordingly, the background will be better represented at any rate exceeding the lossless rate of the ROI. 


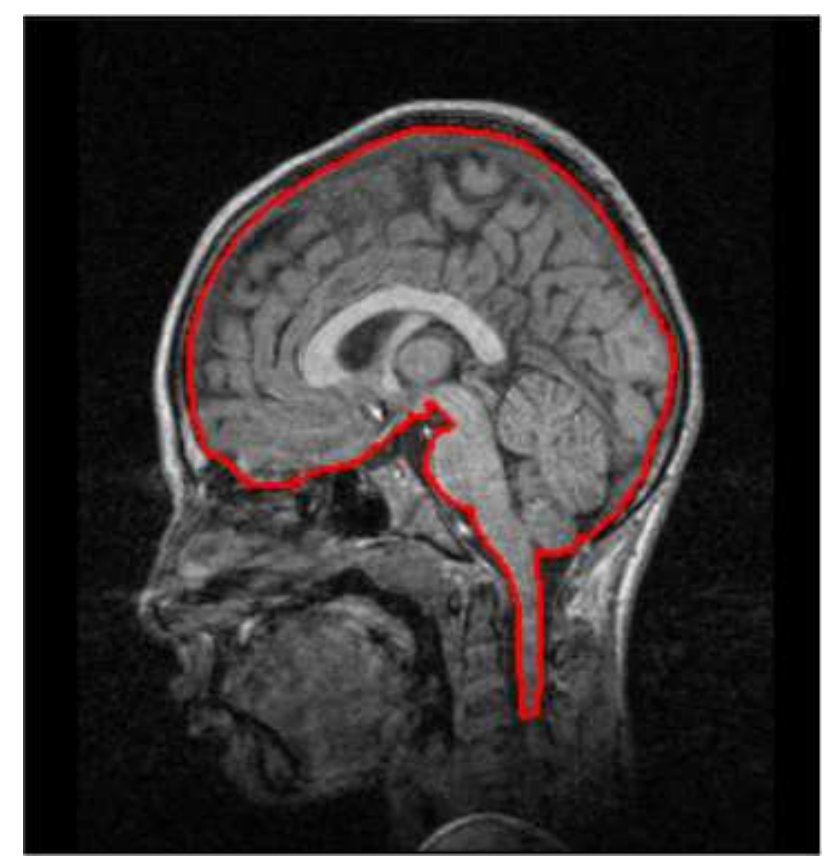

(a)

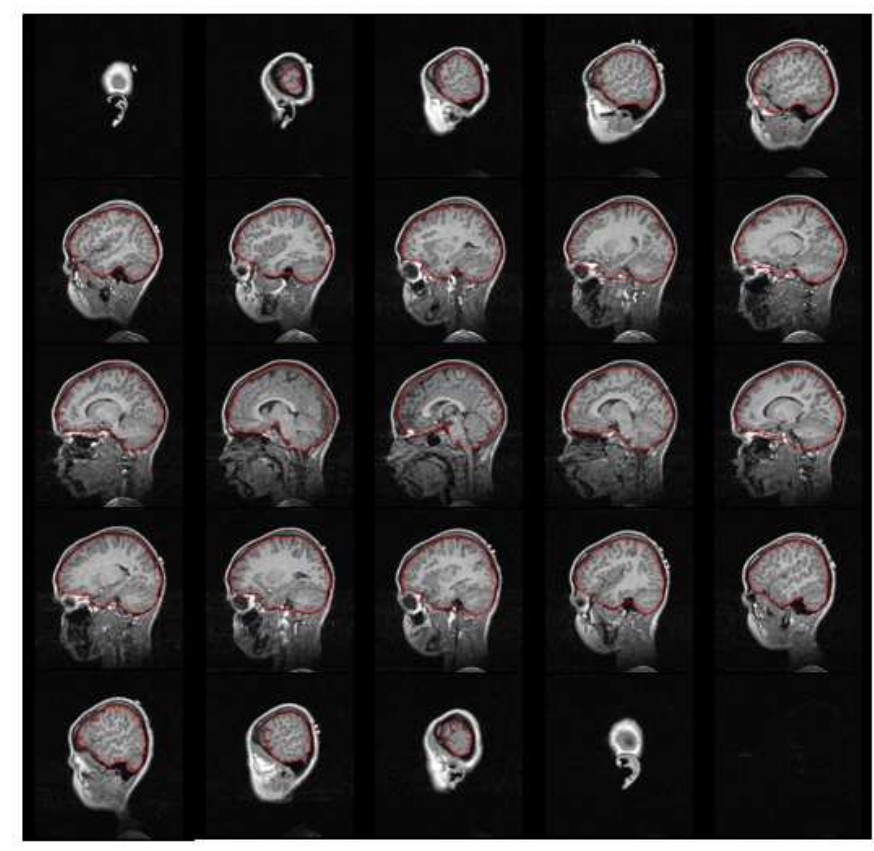

(c)

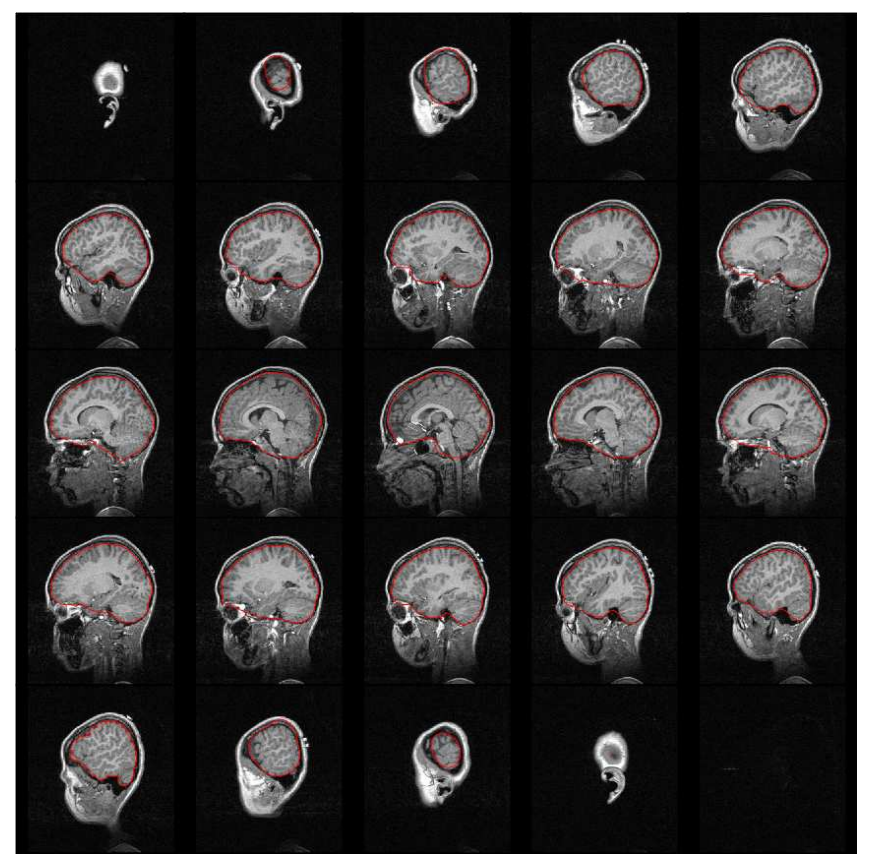

(b)

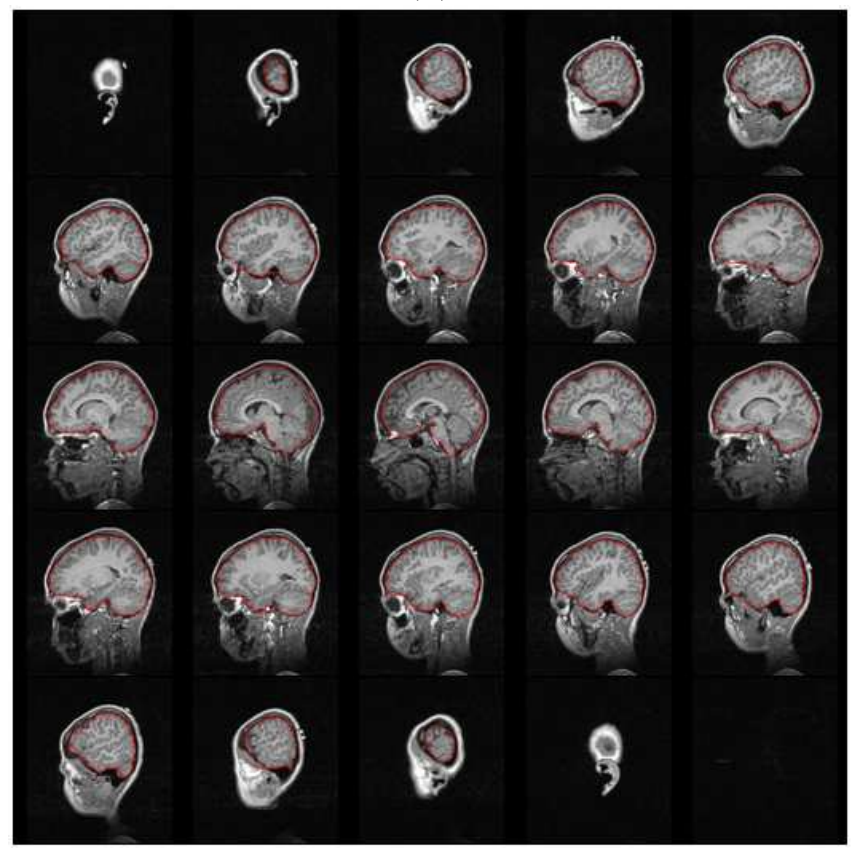

(d)

Figure 4. Results for Dataset 1: (a) atlas; (b) ME; (c) ABME; (d) ABJE. 


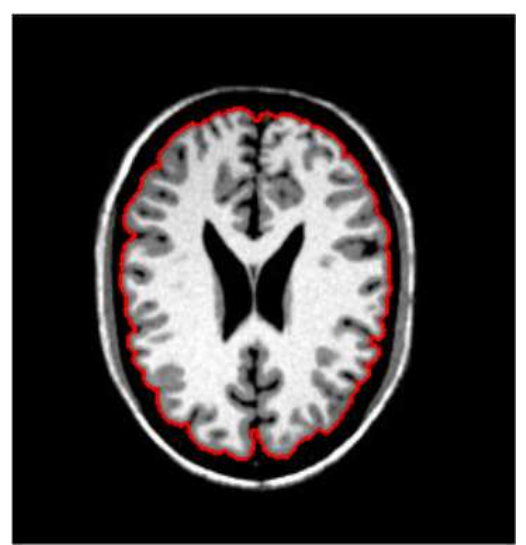

(a)

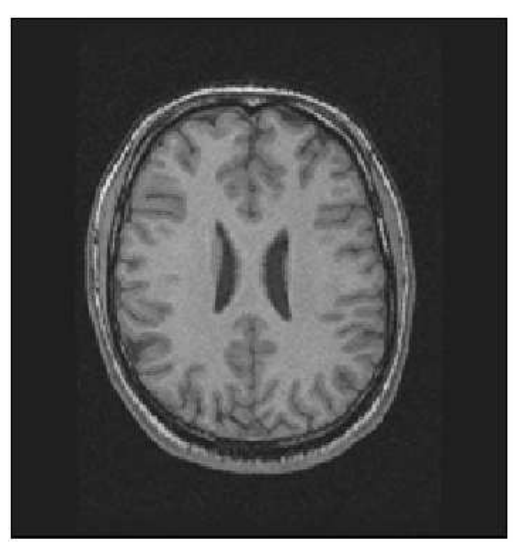

(b)

Figure 5. Dataset 2. (a) Atlas for Dataset 2; (b) Slice n. 75 in the patient's volume. The process start from the image in (b), the most similar to the image selected as atlas.

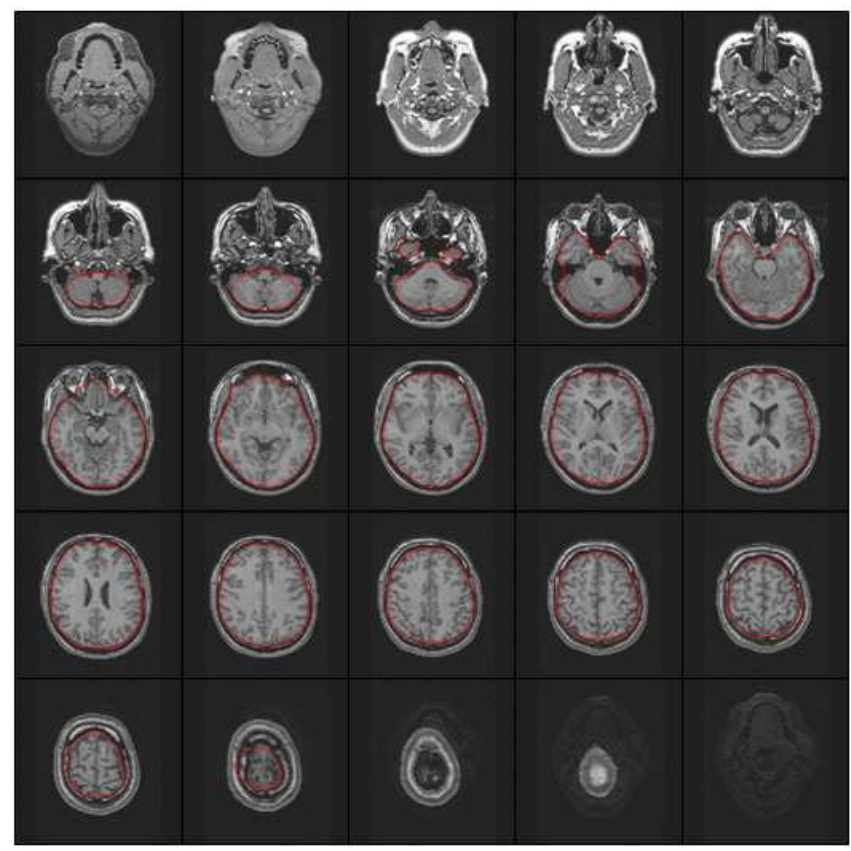

(a)

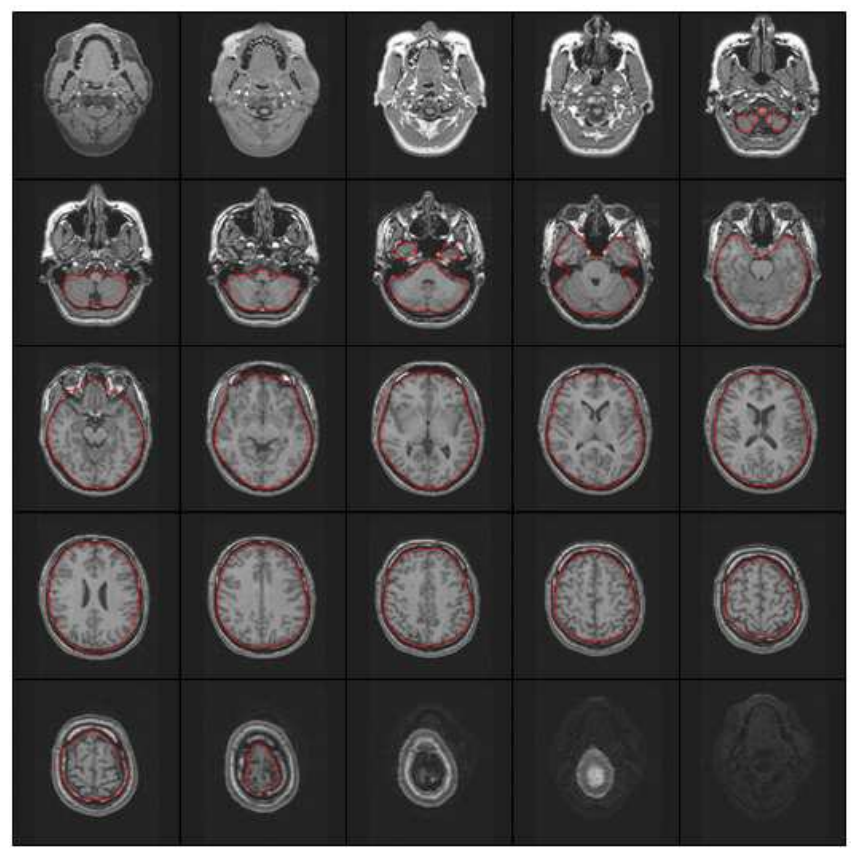

(b)

Figure 6. Dataset 2: (a) ABME; (b) ABJE. 


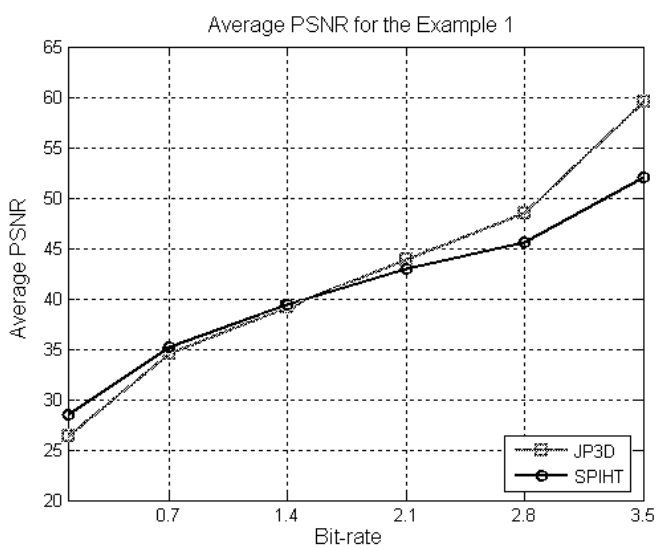

(a)

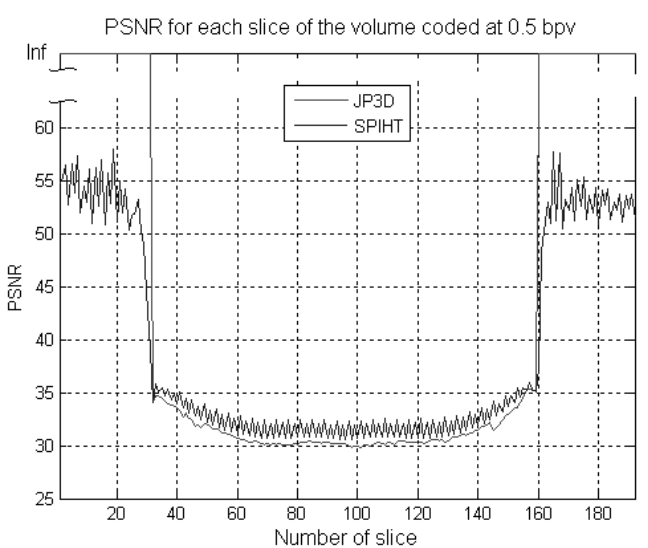

(b)

Figure 7. Dataset 1: (a) PSNR as function of the bit-rate, (b) PSNR as a function of slice number at $0.5 \mathrm{bpv}$.

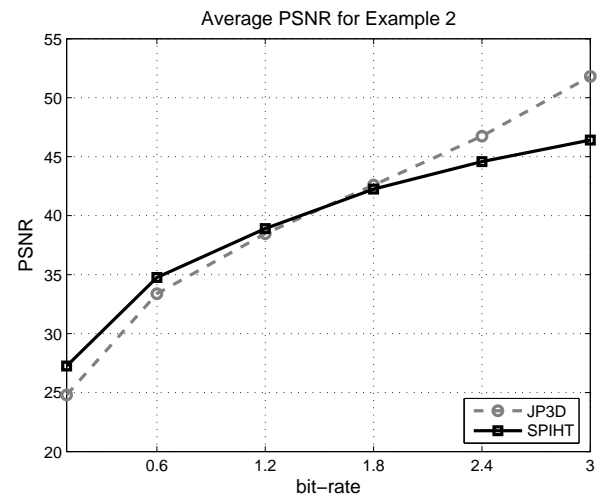

(a)

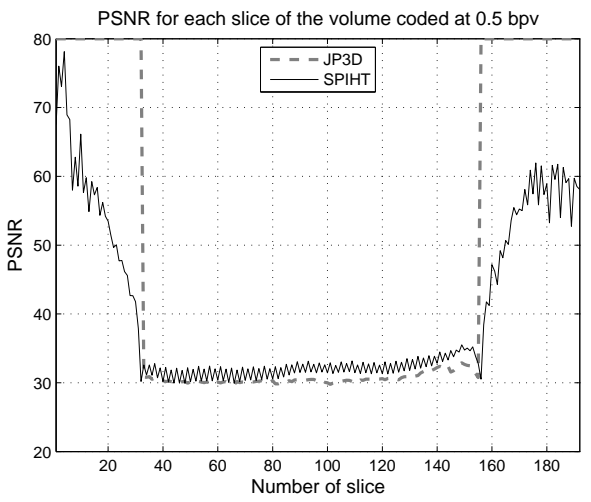

(b)

Figure 8. Dataset 2: (a) PSNR as function of the bit-rate; (b) PSNR for each slice of the volume when decoded at 0.5 bpv.

For Dataset 2, the lossless rates of the entire volume 3.41 and $3.87 \mathrm{bpv}$ for JP3D and 3D-SPIHT, respectively. Figure 8 shows two results in lossy mode on the entire volume. The first plot shows the PSNR as function of the bit-rate for both JP3D and SPIHT; the second gives the PSNR for each slice of the volume when decoded at $0.5 \mathrm{bpv}$. The ROI lossless rates are 1.04 and $1.29 \mathrm{bpv}$. Similar considerations as for Dataset 1 hold.

\section{CONCLUSIONS}

In this work we presented a complete toolbox for automatic segmentation and coding of medical volumetric data. Good segmentation accuracy is reached by the 3D extension of the three considered segmentation methods integrating the active contours and atlas-based approach. An interactive modality is enabled through the design of a graphical interface allowing the medical doctor to reshape the segmentation results at any point in the volume, if needed. High compression efficiency without sacrificing the diagnostically relevant information was proven by encoding two head MRI datasets with the new JP3D standard and the 3D-SPIHT in the ROI based modality. This enables lossless coding of the ROI and the lossy representation of the background. The proposed system is well suited for the integration in PACS. Among the main future developments is the evaluation of its usability (for both segmentation and coding tasks) in the clinical environment. 


\section{REFERENCES}

1. A. Herbulot, S. Jehan-Besson, M. Barlaud, and G. Aubert, "Information theory for image segmentation using shape gradient," LABORATOIRE I3S , 2004.

2. V. Duay, S. Luti, J.-P. Thiran, and G. Menegaz, "Atlas-based active contours: the information theoretic approach," in preparation.

3. 5. The software module is developed by Tim Bruylants. It is an implementation of ISO/IEC 15444-10. Copyright (C) 2006 Vrije Universiteit Brussel (VUB) - Interdisciplinary institute for BroadBand Technology $(I B B T)$

4. Said and W. A. Pearlman, "A new fast and efficient image codec based on set partitioning in hierarchical trees," IEEE, Trans. Circuits Syst. Video Technol., Vol. 6, pp. 243-250, 1996.

5. J. E. Fowler, QCCPACK, Version 0.52, 05-may-2006, Copyright (C) 1997-2006.

6. A. Calderbank, I. Daubechies, W. Sweldens, and B. Yeo, "Lossless image compression using integer to integer wavelet transforms," pp. 596-599, 1997.

7. J. Reichel, G. Menegaz, and M. Nadenau, "Integer wavelet decomposition for lossy image compression," in Proc. of SPIE, 3808, pp. 257-268, (Denver, Colorado), July 18-23 1999.

8. J. Reichel, G. Menegaz, M. Nadenau, and M. Kunt, "Integer wavelet transform for embedded lossy to lossless image compression," IEEE Trans. on Image Processing 10, pp. 383-392, March 2001. 